\section{ORIGINAL RESEARCH}

E. Canu

F. Agosta

N. Riva

S. Sala

A. Prelle

D. Caputo

M. Perini

G. Comi

M. Filippi

\title{
The Topography of Brain Microstructural Damage in Amyotrophic Lateral Sclerosis Assessed Using Diffusion Tensor MR Imaging
}

BACKGROUND AND PURPOSE: ALS leads to macrostructural (ie, cortical atrophy and hyperintensities along the corticospinal tract) and microstructural (ie, gray matter intrinsic damage) central nervous system abnormalities. We used a multimodal voxelwise imaging approach to assess microstructural changes independent of macrostructural volume loss in patients with ALS compared with HCs.

MATERIALS AND METHODS: Twenty-three patients with ALS and $14 \mathrm{HCs}$ were studied. Conventional imaging and DTI were performed. Images were processed by using SPM5 to assess measures of gray and white matter atrophy as well as microstructural damage (ie, MD and FA). DTI alterations independent of volume loss were investigated.

RESULTS: When we accounted for both gray and white matter atrophy, patients with ALS showed increased MD values in several gray and white matter areas mainly located in the orbitofrontal and frontotemporal regions bilaterally, in the right genu of the corpus callosum, and in the right posterior limb of the internal capsule. When we accounted for white matter volume loss, patients with ALS showed decreased FA along the corticospinal tract bilaterally and in the left inferior frontal lobe relative to HCs. The MD of the orbitofrontal regions bilaterally was associated significantly with disease duration

CONCLUSIONS: In patients with ALS, DTI detects microstructural changes independent of brain tissue loss. The affected regions included both motor and extramotor areas. The extent of ALS-related DTI abnormalities was greater than that disclosed by the volumetric analysis.

ABBREVIATIONS: ALS = amyotrophic lateral sclerosis; $\mathrm{ANCOVA}=$ analysis of covariance; $\mathrm{B} 0=\mathrm{DTI}$ with no diffusion weighting; $\mathrm{BA}=$ Brodmann area; $\mathrm{BPM}=$ biological parametric mapping; DARTEL = Diffeomorphic Anatomical Registration using Exponentiated Lie algebra registration method; DTI = diffusion tensor imaging; FA = fractional anisotropy; FLAIR = fluid-attenuated inversion recovery; $\mathrm{FWHM}=$ full width at half maximum; $\mathrm{GM}=$ gray matter; $\mathrm{HC}=$ healthy control; LL- and UL-MRC = lower limb- and upper limb-Medical Research Council; MD = mean diffusivity; $\mathrm{MNI}=$ Montreal Neurological Institute; MRC = Medical Research Council scale; r-ALSFRS = ALS Functional Rating Scale-Revised; SOD1 = superoxide dismutase; SPM = statistical parametric mapping; $\mathrm{T} 1 \mathrm{w}=\mathrm{T} 1$-weighted; $\mathrm{T} 2 \mathrm{w}=\mathrm{T} 2$-weighted; $\mathrm{UMN}=$ upper motor neurons; $\mathrm{VBM}=$ voxel-based morphometry; $\mathrm{WM}=$ white matter

$\mathbf{A}^{\mathrm{L}}$ LS is a progressive neurodegenerative disease, which is associated with heterogeneous pathologic features. The main pathologic abnormalities in patients with ALS include loss of motor neurons in the primary motor cortex, brain stem, and spinal anterior horn, as well as axonal degeneration, demyelination, and reactive gliosis of the corticospinal tract. ${ }^{1}$ Such changes can result in gross abnormalities such as atrophy of the motor/premotor cortex and macroscopic lesions along the corticospinal tract, which can be seen on conventional MR imaging of the brain. ${ }^{2}$ Although the etiology of ALS remains unknown, mutations in the gene encoding $\mathrm{Cu} / \mathrm{Zn}$-SOD1 have

Received August 19, 2010; accepted after revision October 30

From the Neuroimaging Research Unit (E.C., F.A., S.S., M.F.) and Department of Neurology (N.R., G.C., M.F.), Institute of Experimental Neurology, Division of Neuroscience, Scientific Institute and University Ospedale San Raffaele, Milan, Italy; Ospedale Fatebenefratelli e Oftalmico (A.P.), Milan, Italy; Department of Neurology (D.C.), Scientific Institute Fondazione Don Gnocchi, Milan, Italy; and Department of Neurology (M.P.), Ospedale di Gallarate, Gallarate, Italy.

Please address correspondence to Massimo Filippi, MD, Neuroimaging Research Unit, Institute of Experimental Neurology, Division of Neuroscience, Scientific Institute and University Ospedale San Raffaele, Via Olgettina, 60, 20132 Milan, Italy; e-mail: m.filippi@hsr.it

Indicates article with supplemental on-line figure.

DOI 10.3174/ajnr.A2469 been implicated in a fraction of familial cases of the disease. ${ }^{3}$ Emerging research has demonstrated that mutations and pathology associated with the TAR DNA binding protein-43 gene and protein may be more common than SOD1 mutations in familial and sporadic ALS. ${ }^{3}$ Putative mechanisms of toxicity targeting motor neurons include oxidative damage, accumulation of intracellular aggregates, mitochondrial dysfunction, defects in axonal transport, growth factor deficiency, aberrant RNA metabolism, glial cell pathology, and glutamate excitotoxicity. Convergence of these pathways is likely to mediate disease onset and progression. ${ }^{3}$

DTI allows measuring the random diffusional motion of water molecules and, thus, provides quantitative indices of the structural and orientation features of central nervous system tissues. ${ }^{4} \mathrm{MD}$ is a measure of the magnitude of diffusion, whereas FA is a measure of the directionality of water diffusion. ${ }^{4,5}$ Alterations in the brain microstructure associated with ALS have the potential to modify water diffusion characteristics, which can be reflected in changes of MD and FA. DTI studies in patients with ALS showed consistently decreased FA and increased MD values along the corticospinal tract. ${ }^{6}$ Decreased FA and increased MD have also been shown in the corpus callosum ${ }^{7-11}$ and in the gray and white matter of non- 
motor frontal ${ }^{8,10-12}$ and temporal ${ }^{10-12}$ regions of patients with ALS compared with controls.

The meaning of MD and FA changes in ALS, however, must be interpreted cautiously because they can be influenced by gray and white matter volume loss. ${ }^{13}$ For instance, white matter diffusivity abnormalities are related to several aspects of the white matter microstructure, some of which are likely to also affect the volumetry of the damaged areas (eg, the degree of myelination and axonal degeneration). ${ }^{4,5}$ Using VBM or measurements of cortical thickness, previous studies in patients with ALS showed atrophy of the primary motor and premotor cortices as well as of several other frontal and temporal regions. ${ }^{7,12,14-18}$ White matter volume reductions too have been found in motor and nonmotor regions of patients with ALS. ${ }^{16,19,20}$ Recently, the ability of DTI to recognize brain microstructural alterations, regardless of the presence of volume loss, in healthy subjects ${ }^{21}$ and patients with Alzheimer disease has been shown. ${ }^{22}$ These observations suggest that microstructural tissue abnormalities may precede gray and white matter volume loss in target regions typically hit by normal aging or Alzheimer disease. ${ }^{21,22}$

Against this background, we wished to assess whole brain $\mathrm{MD}$ and FA alterations in patients with ALS compared with controls by using a voxelwise method, which takes into account volume differences on a voxel-per-voxel basis. ${ }^{23}$

\section{Materials and Methods}

The study was conducted with institutional review board approval. Written informed consent was obtained from each participant.

\section{Subjects}

Twenty-three patients with probable or definite ALS, ${ }^{24}$ and mild disability, defined as a score $\geq 20$ on the r-ALSFRS, ${ }^{25}$ were included (Table 1). Three patients had bulbar-onset disease. None of the patients had clinical evidence of frontotemporal dementia. Experienced neurologists, unaware of the MR imaging results, administered the r-ALSFRS questionnaire within 48 hours of acquisition of the MR images. Muscle strength was scored by using the MRC scale from 0 to $5 .{ }^{26} \mathrm{UMN}$ involvement was assessed by totaling the number of pathologic UMN signs on examination (maximum total score $=16$ ). ${ }^{27}$ Fourteen sex- and age-matched healthy individuals, with no history of neurologic or psychiatric disorders, served as controls.

\section{MR Imaging Acquisition}

Conventional and DTI scans were acquired by using a $1.5 \mathrm{~T}$ system (Avanto; Siemens, Erlangen, Germany). The following pulse sequences were obtained: 1 ) dual-echo turbo spin-echo ( $\mathrm{TR}=2650 \mathrm{~ms}$, first-echo $\mathrm{TE}=28 \mathrm{~ms}$, second-echo $\mathrm{TE}=113 \mathrm{~ms}$, echo-train length $=5$, number of sections $=50$, section thickness $=2.5 \mathrm{~mm}$ with no gap, matrix size $=512 \times 512$, FOV $\left.\left.=250 \mathrm{~mm}^{2}\right) ; 2\right)$ FLAIR $(\mathrm{TR}=$ $10,000 \mathrm{~ms}, \mathrm{TE}=115 \mathrm{~ms}, \mathrm{TI}=2500 \mathrm{~ms}$, flip angle $=150^{\circ}$, number of sections $=50$, section thickness $=2.5 \mathrm{~mm}$ with no gap, matrix size $=$ $256 \times 224$, FOV $=240 \times 210 \mathrm{~mm}^{2}$ ); 3) sagittal 3D T1-weighted magnetization-prepared rapid acquisition of gradient echo $(\mathrm{TR}=$ $2,000 \mathrm{~ms}, \mathrm{TE}=3.93 \mathrm{~ms}$, flip angle $=128^{\circ}, \mathrm{FOV}=270 \mathrm{~mm}^{2}$, matrix size $=256 \times 256$, voxel size $=0.9 \times 0.5 \times 0.5 \mathrm{~mm}^{3}$, slab thickness $=$ $187.2 \mathrm{~mm})$; and 4) pulsed gradient spin-echo echo-planar (TR = $6500 \mathrm{~ms}, \mathrm{TE}=95 \mathrm{~ms}$, number of sections $=40$, section thickness $=$ $2.5 \mathrm{~mm}$ with no gap, matrix size $\left.=128 \times 128, \mathrm{FOV}=240 \mathrm{~mm}^{2}\right)$ with

\begin{tabular}{lccc}
\hline $\begin{array}{l}\text { Table 1: Sociodemographic and clinical features of HCs and } \\
\text { patients with } \text { ALS }^{\mathbf{a}}\end{array}$ & \multicolumn{3}{l}{} \\
\hline \multicolumn{4}{c}{ Healthy } \\
& Controls & Patients with ALS & $P^{\text {a }}$ \\
\hline No. & 14 & 23 & \\
Age (yr) & $60 \pm 11(43-76)$ & $61 \pm 10(40-78)$ & .84 \\
Women & $8(57 \%)$ & $11(48 \%)$ & .74 \\
r-ALSFRS score & & $33.0 \pm 6.8(22-46)$ & \\
Disease duration (mo) & & $24.4 \pm 18.6(3-72)$ & \\
Progression rate & & $1.0 \pm 1.0(0.2-3.7)$ & \\
UL-MRC score & & $56.6 \pm 11.0(39-70)$ & \\
LL-MRC score & & $35.9 \pm 13.8(2-50)$ & \\
UMN score & & $13.2 \pm 3.9(3-16)$ & \\
\hline
\end{tabular}

a $T$ test or Pearson $\chi^{2}$ test as appropriate. Values are mean \pm SD (range) and No. (percentage). Progression rate was calculated as follows: (48 - r-ALSFRS score) / months of disease duration.

diffusion-encoding gradients applied in 12 noncollinear directions $(b$ $=1000 \mathrm{~s} / \mathrm{mm}^{2}$, number of averages $=8$ ).

\section{MR Imaging Analyses}

All MR imaging analyses were performed by a single experienced observer blinded to clinical findings. Corticospinal tract hyperintensities were identified on dual-echo and FLAIR scans.

VBM. VBM was performed by using SPM5 (Wellcome Department of Imaging Neuroscience, London, United Kingdom) and the DARTEL registration method, ${ }^{28}$ as previously described. ${ }^{22}$ Briefly, the process was as follows: 1) T1-weighted images were segmented by using VBM5.1 toolbox (available at: http://dbm.neuro.uni-jena.de/ beta-version-of-vbm51-toolbox/ $)^{29}$ to produce tissue probability maps in the MNI space. 2) The images were imported in DARTEL, 3) rigidly aligned, and segmented into gray and white matter (by using the segmentation parameters from the first step) and resampled to 1.5 -mm isotropic voxels. 4) The gray and white matter segments were coregistered simultaneously by using the fast diffeomorphic image registration algorithm and the flow fields were created. ${ }^{28} 5$ ) The flow fields were then applied to the rigidly aligned segments to warp them to the common DARTEL space and then were modulated by using the Jacobian determinants. 6) The modulated images from DARTEL were normalized to the MNI template by using an affine transformation estimated from the DARTEL gray matter template and the a priori gray matter probability map without resampling (http://brain map.wisc.edu/pages/8-Normalizing-DARTEL-Templates-to-MNISpace). ${ }^{30}$ Before the statistical computations, the images were smoothed with an 8-mm FWHM Gaussian filter (see Fig 1 as a flow chart of the procedure)

DTI Analyses. DTI analysis was performed by using in-house software. Diffusion-weighted images were first corrected for distortion induced by eddy currents ${ }^{31}$; then the diffusion tensor was estimated by linear regression ${ }^{5}$ and MD and FA maps were computed. ${ }^{4}$ To use gray and white matter volumes as covariates in the statistical analysis of the DTI data, we had to align the FA and MD maps with the T1-weighted images normalized by using DARTEL as previously reported. ${ }^{22}$ Briefly, the process was the following: a) The nondiffusionweighted (B0) images were normalized to the MNI T2 template in SPM5. b) A custom FA template was created by normalizing the FA images by using the normalization parameters from step a; these images were then averaged and smoothed with an 8-mm FWHM Gaussian filter. c) The custom FA template was normalized to the custom T1-weighted template. d) Native space FA and MD images were normalized to the custom FA template (from step c). e) The normaliza- 


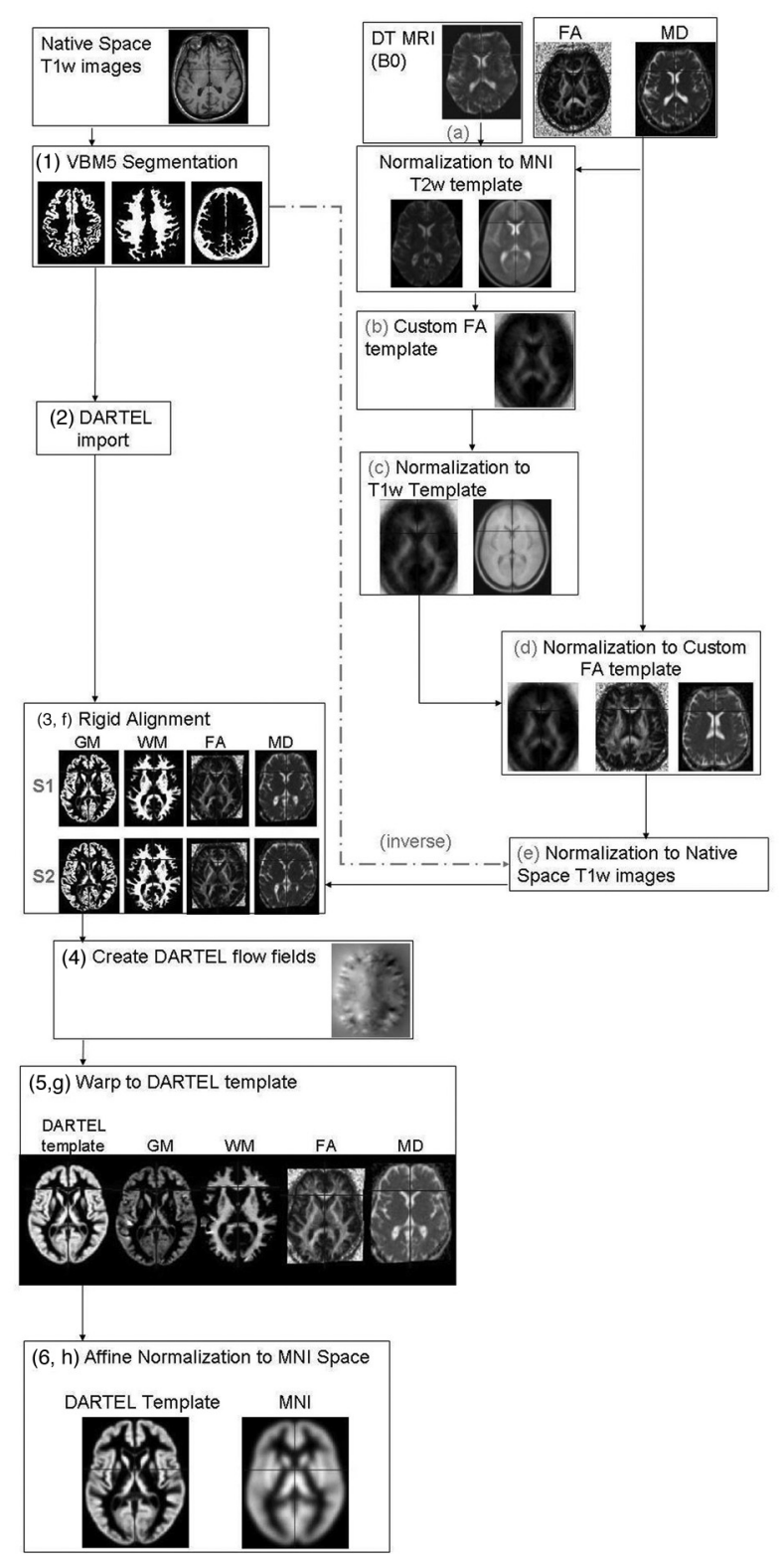

Fig 1. Flow chart of the image processing streams. The text provides a description of the image-processing stream with numbers referring to T1-weighted processing and letters to DTI processing.

tion parameters from step d were multiplied by the inverse transformation parameters of the T1-weighted segmentation (VBM step 1) and applied to both the FA and MD images. f) FA and MD images were rigidly aligned by using the rigid-body component of the transformations produced in VBM step 1 and resampled to $1.5-\mathrm{mm}$ isotropic voxels. g) They were warped by using flow fields from DARTEL (VBM step 4), but they were not modulated. h) DARTEL FA and MD images were normalized to the MNI space by using the affine transformation estimated from the DARTEL gray matter template and the a priori gray matter probability map in SPM5 (VBM step 5 without resampling; http://brainmap.wisc.edu/normalizeDARTELtoMont real Neurologic Institute). FA and MD images were smoothed with an 8-mm full FWHM Gaussian filter (see Fig 1 as a flow chart of the procedure).

\section{Statistical Analysis}

Between-group differences in gray and white matter volumes and DTI measures, regardless of the effect of the other imaging technique, were performed by using SPM5. The same models were used to compare MR imaging changes between patients with and without hyperintense lesions along the corticospinal tracts. Next, microstructural (MD and FA) group differences were tested by using macrostructural variables (gray and white matter volumes) as voxelwise covariates by using the BPM toolbox implemented in SPM5. ${ }^{23}$ This approach tests for locations where DTI data explain unique variance not accounted for by gray or white matter volume. Reverse ANCOVA comparisons assessing macrostructural volume (gray and white matter volumes) differences between patients and HCs independent of microstructural alterations (FA and MD) were also performed. Finally, multiple regressions were performed in SPM5 to investigate the relationship between MD and FA alterations and clinical findings. All VBM results were obtained by using the general linear model, and the significance of each effect was determined with the theory of Gaussian fields. ${ }^{29}$ Between-group comparisons and correlation analyses were assessed by using an uncorrected statistical threshold of $P<.001$ or $P<.005$, in at least 20 contiguous voxels.

Gray and White Matter Volume Loss with and without Accounting for Microstructural Abnormalities. Two ANCOVAs were computed to assess gray and white matter volume differences in patients with ALS versus HCs. Smoothed gray and white matter images entered 2 separate models as factors, and total intracranial volume was included as a covariate. Two ANCOVAs were also computed by using the BPM toolbox ${ }^{23}$ to assess gray and white matter differences, independent of microstructural alterations, between patients with ALS and HCs. Smoothed gray and white matter maps were entered as dependent variables in 2 separate models. Smoothed and modulated $\mathrm{MD}$ and FA images were included in the models as imaging covariates.

MD and FA Alterations with and without Accounting for Gray and White Matter Volume Loss. Two ANCOVAs were computed to assess MD and FA differences in patients with ALS versus HCs. Smoothed MD and FA entered the models as factors. Two further ANCOVAs were computed by using the BPM toolbox ${ }^{23}$ to assess MD and FA differences between patients with ALS and HCs. Smoothed MD and FA images were entered as dependent variables in 2 separate models. Smoothed and modulated gray and white matter segmented maps were included in the models as imaging covariates.

The Relationship between MR Imaging and Clinical Variables. Patients with and without hyperintense lesions along the corticospinal tracts were compared in terms of clinical variables by using an ANCOVA adjusted for the subject's age. We performed multiple regressions in which the independent variables were the clinical measures and the dependent variable were subject's MD and FA maps, as well as gray and white matter volume maps. Analyses were restricted to the significant clusters obtained from the ANCOVAs assessed by using BPM.

\section{Results}

On the dual-echo and FLAIR scans, hyperintensities of the corticospinal tract were detected bilaterally in 12 (52\%) patients with ALS and in 5 (35\%) HCs. The remaining 11 patients did not show any brain hyperintensity. 


\begin{tabular}{|c|c|c|c|c|c|c|}
\hline \multirow[b]{2}{*}{ Cluster Size $^{a}$} & \multirow[b]{2}{*}{ BA } & \multirow[b]{2}{*}{ Regions } & \multicolumn{3}{|c|}{ MNI Coordinates (mm) } & \multirow[b]{2}{*}{$T$ Values } \\
\hline & & & $X$ & $Y$ & Z & \\
\hline \multicolumn{7}{|c|}{ Gray matter volume loss } \\
\hline 54 & BA 39 & $\mathrm{R}$ angular gyrus & 33 & -57 & 45 & 4.28 \\
\hline 29 & BA 4 & L precentral gyrus & -45 & -6 & 30 & 4.02 \\
\hline 65 & BA 46 & L middle frontal gyrus & -24 & 40 & 19 & 4.00 \\
\hline \multirow[t]{2}{*}{46} & BA 4 & R precentral gyrus & 30 & -16 & 41 & 3.72 \\
\hline & BA 4 & R precentral gyrus & 38 & -10 & 43 & 3.43 \\
\hline 22 & BA 48 & $\mathrm{R}$ rolandic operculum & 56 & 2 & 14 & 3.62 \\
\hline \multicolumn{7}{|c|}{ White matter volume loss } \\
\hline 51 & & L superior frontal lobe & -19 & 53 & 28 & 4.14 \\
\hline \multirow[t]{3}{*}{240} & & R supplementary motor area & 8 & -26 & 54 & 3.92 \\
\hline & & $\mathrm{R}$ precentral region & 17 & -15 & 70 & 3.61 \\
\hline & & $\mathrm{R}$ precentral region & 16 & -20 & 59 & 3.57 \\
\hline 47 & & L supplementary motor area & -3 & -21 & 56 & 3.80 \\
\hline 25 & & L inferior temporal area & -40 & -28 & -13 & 3.59 \\
\hline
\end{tabular}

a The number of voxels. $P<.001$ uncorrected within at least 20 contiguous voxels.

Gray and White Matter Volume Loss with and without Accounting for Microstructural Changes

Compared with HCs, patients with ALS showed significant gray matter volume losses in the precentral gyrus bilaterally, right rolandic operculum and angular gyrus, and left middle frontal gyrus $(P<.001$ uncorrected; Table 2 and Fig 2$)$. In patients with ALS, significant volume losses were also detected in the following white matter areas: left superior frontal region, the vicinities of the supplementary motor area bilaterally, right precentral region, and left inferior temporal region $(P<.001$ uncorrected; Table 2 and Fig 2$)$. No volumetric difference was found between patients with and without hyperintense lesions along the corticospinal tracts. When we controlled for MD and FA values, patients with ALS relative to HCs showed a small region of gray matter loss in the right angular gyrus (cluster size $=28$ voxels; MNI coordinates: $\mathrm{x}=$ $33, \mathrm{y}=-57, \mathrm{z}=45 ; t$ value $=4.04)$. No regions of white matter loss were found in patients with ALS compared with HCs after accounting for microstructural abnormalities.

\section{MD and FA Alterations with and without Accounting for Gray and White Matter Volume Loss}

After controlling for both gray and white matter volume loss, patients with ALS showed significant increased MD values in the precentral gyrus bilaterally, left inferior frontal gyrus, left insula, left middle temporal gyrus, right temporal pole, left postcentral gyrus, right putamen, right angular gyrus, left supramarginal gyrus, orbitofrontal white matter bilaterally, right posterior limb of the internal capsule, right genu of the corpus callosum, right middle occipital white matter, and the anterior lobe of the cerebellum bilaterally $(P<.001$ uncorrected; Table 3 and Fig 3). When we controlled for gray and white matter volume loss, patients with ALS relative to HCs showed significantly decreased FA values in the precentral and premotor white matter regions bilaterally and the left inferior frontal white matter $(P<.001$ uncorrected; Table 4 and Fig 3$)$. Similar results were obtained without imaging covariates (Online Figure).

Relationship between MR Imaging and Clinical Variables No clinical difference was found between patients with and without hyperintense lesions along the corticospinal tracts. In patients with ALS, MD of the orbitofrontal region bilaterally was significantly related to disease duration (MNI coordinates: $\mathrm{x}=14, \mathrm{y}=45, \mathrm{z}=-13 ; r=0.75, P<.001$ uncorrected; MNI coordinates: $\mathrm{x}=-18, \mathrm{y}=43, \mathrm{z}=-12 ; r=0.60, P<$ .005 uncorrected). No correlation was found between DTI measures and other clinical variables. Gray and white matter volume losses did not correlate with clinical measures.

\section{Discussion}

In this study, we used a multimodal voxelwise imaging approach $^{23}$ to assess microstructural damage independent of gross gray and white matter volume loss in patients with ALS with mild disability. We found that DTI can detect microstructural brain abnormalities in ALS, even after correcting for atrophic changes. The affected regions included both motor and extramotor areas. Furthermore, the extent of ALS-related DTI abnormalities was greater than that disclosed by the VBM analysis. As a consequence, our findings suggest that DTI abnormalities are more sensitive than volumetry to alterations of brain tissue integrity associated with ALS. There are at least 2 possible explanations, which are not mutually exclusive, for such a finding. First, diffusivity metrics may be sensitive to pathologic changes that are too subtle to have an impact on volume measurements. Second, diffusivity abnormalities may precede atrophy, which appears only after a significant amount of intrinsic tissue damage has occurred. All of this calls for longitudinal studies to clarify the multifaceted relationship between gray and white matter volume and microstructural changes in ALS and suggests that DTI should be viewed as a promising approach to improve our understanding of this condition.

In agreement with previous pathologic ${ }^{1}$ and neuroimaging studies, ${ }^{7,12,14-18}$ gray matter loss in the precentral regions bilaterally and in the right middle frontal gyrus was found in patients with ALS. The pattern of white matter loss, which included precentral/premotor regions, the tissue in the vicinity of the supplementary motor area, as well as the frontal and temporal lobes, was also previously observed. ${ }^{16,19,20}$ Although pathologic studies reported the presence of inflammatory infiltrates in the ALS brain, ${ }^{32}$ this is not associated with macroscopic alterations such as lesions or edema. As a consequence, we do not believe that inflammation affected our volumetric 


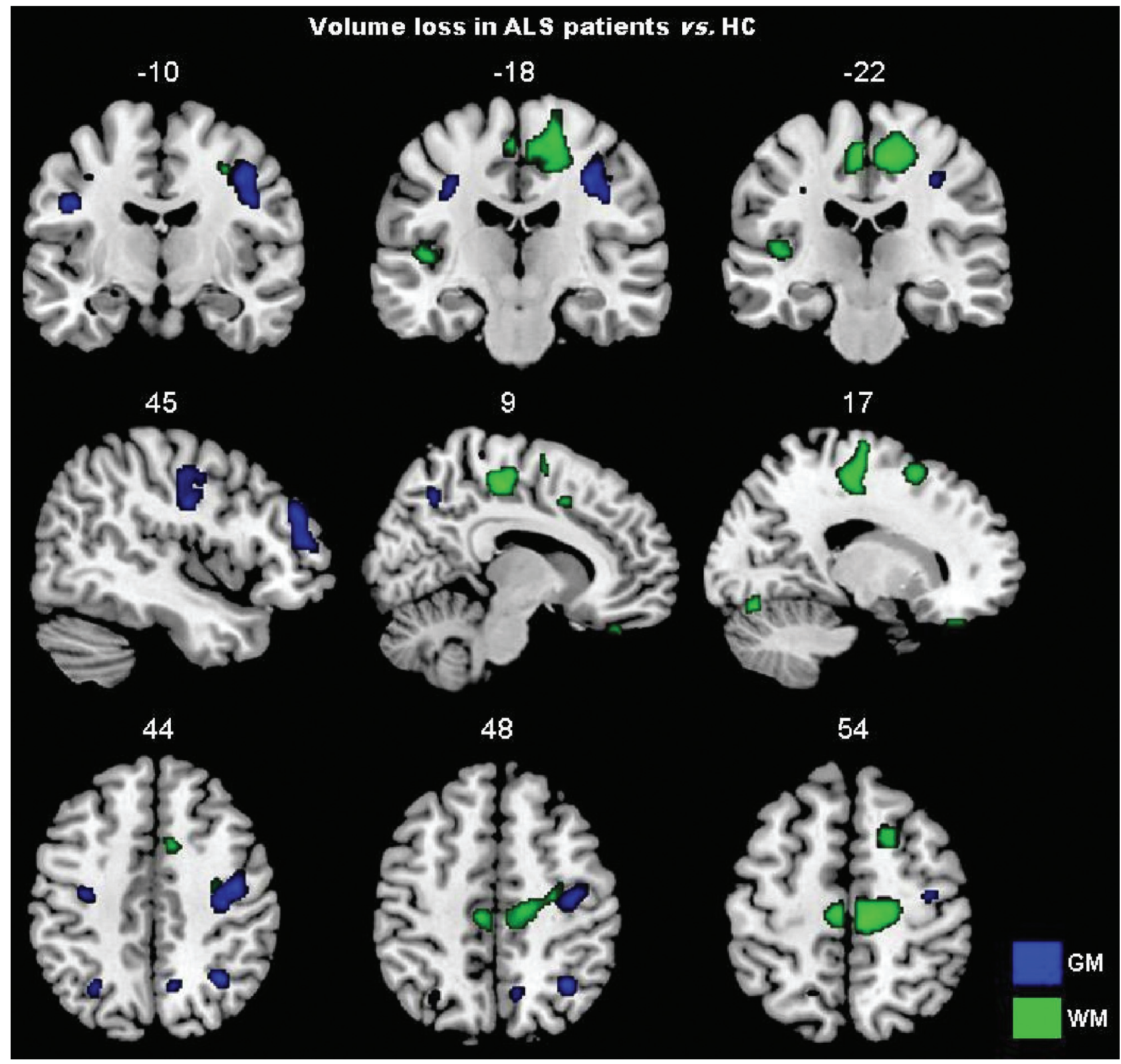

Fig 2. Gray and white matter volume loss in patients with ALS compared with $\mathrm{HCs}$ is displayed on the same MNI template. For illustrative purposes, the contrasts are shown at a threshold of $P<.005$ uncorrected in at least 20 contiguous voxels. Results are in neurologic convention (right is right).

results at a great deal. The comparison of MD and FA values between patients and HCs without gray and white matter volume maps as imaging covariates showed a much more widespread pattern of gray and white matter microstructural changes, which resemble closely those reported in previous DTI studies of ALS. ${ }^{6,7,11,12}$ Patients had an increased MD not only in the precentral gyri and along the corticospinal tract bilaterally but also in the corpus callosum; several bilateral frontal, temporal, and parietal regions; and the right putamen and anterior lobe of the cerebellum bilaterally. Patients with ALS also showed decreased FA values in white matter regions located in the vicinities of motor and premotor cortices, which again fit with previous findings. ${ }^{7,8,10-12}$ No difference in volumetric and DTI features, as well as in clinical variables, was found between patients with ALS with and without corticospinal hyperintensities on conventional MR images. These findings are in line with previous MR imaging studies in ALS showing a low sensitivity and specificity of such abnormalities. , $^{\text {,33-37 }}$

When DTI findings were controlled for gray and white matter volumes, MD and FA differences between patients and HCs were very similar to those obtained without correction, thus suggesting that DTI detects ALS-related microstructural changes independent of brain tissue loss. Compared with the analysis in which MD values were controlled for volume loss, the analyses without correction showed only an additional cluster located in the left internal capsule. This finding suggests that most microstructural alterations that we and others observed in patients relative to HCs are reflecting intrinsic tissue damage and not volumetric differences of the regions studied. Several pathologic substrates are likely to contribute to the observed abnormalities of diffusion parameters in the 


\begin{tabular}{|c|c|c|c|c|c|c|}
\hline \multirow[b]{2}{*}{ Cluster Size $^{\mathrm{a}}$} & \multirow[b]{2}{*}{ BA } & \multirow[b]{2}{*}{ Regions } & \multicolumn{3}{|c|}{ MNI Coordinates (mm) } & \multirow{2}{*}{$\begin{array}{c}T \\
\text { Values }\end{array}$} \\
\hline & & & $x$ & Y & Z & \\
\hline 59 & BA6 & $L$ precentral gyrus & -54 & 1 & 26 & 5.29 \\
\hline 64 & \multirow[t]{4}{*}{ BA6 } & $\mathrm{R}$ precentral gyrus & 49 & 2 & 25 & 5.13 \\
\hline 224 & & R putamen & 21 & 17 & -4 & 4.71 \\
\hline & & $\mathrm{R}$ middle orbitofrontal white matter & 17 & 18 & -10 & 3.45 \\
\hline 127 & & L superior orbitofrontal white matter & -18 & 32 & -8 & 4.46 \\
\hline 33 & \multirow[t]{3}{*}{ BA45 } & $L$ inferior frontal gyrus & -45 & 9 & 24 & 4.34 \\
\hline 88 & & $\mathrm{R}$ middle occipital white matter & 38 & -73 & 6 & 4.34 \\
\hline 78 & & R posterior limb of the internal capsule & 16 & -14 & 0 & 4.13 \\
\hline 47 & BA21 & L middle temporal gyrus & -59 & -60 & 1 & 4.10 \\
\hline 28 & BA39 & $\mathrm{R}$ angular gyrus & 40 & -54 & 40 & 4.07 \\
\hline 24 & BA20 & $\mathrm{R}$ inferior temporal pole & 65 & -13 & -23 & 4.06 \\
\hline 41 & BA3 & L postcentral gyrus & -60 & -11 & 31 & 4.02 \\
\hline 23 & \multirow[t]{5}{*}{ BA21 } & $\mathrm{R}$ middle temporal pole & 51 & 12 & -31 & 4.01 \\
\hline 116 & & $\mathrm{R}$ genu of the corpus callosum & 9 & 32 & -1 & 3.98 \\
\hline & & R superior orbitofrontal white matter & 17 & 38 & -4 & 3.63 \\
\hline 108 & & $L$ anterior lobe of the cerebellum & -18 & -25 & -29 & 3.95 \\
\hline & & L middle cerebellar peduncle & -13 & -32 & -29 & 3.90 \\
\hline 20 & BA22 & R superior temporal lobe & 52 & -46 & 22 & 3.91 \\
\hline 38 & BA13 & L insula & -38 & 14 & -1 & 3.90 \\
\hline 55 & \multirow[t]{2}{*}{ BA45 } & $L$ inferior frontal gyrus & -37 & 43 & 11 & 3.76 \\
\hline 23 & & $\mathrm{R}$ anterior lobe of the cerebellum & 16 & -55 & -25 & 3.68 \\
\hline 26 & BA40 & L supramarginal gyrus & -49 & -24 & 32 & 3.58 \\
\hline & BA40 & L supramarginal gyrus & -40 & -24 & 33 & 3.46 \\
\hline
\end{tabular}

${ }^{a}$ The number of voxels. $P<.001$ uncorrected within at least 20 contiguous voxels.

gray and white matter of patients with ALS. ${ }^{4,38}$ The increased content of unhindered isotropically diffusing water in the extracellular spaces (due to axonal and neuronal loss) can lead to an increased average MD. ${ }^{4,38}$ Intracellular abnormalities of the surviving axons along with the presence of cell debris, inflammatory infiltrates, and gliosis, which are not associated with the same anisotropic organization of the normal tissue, can result in a reduced average FA. ${ }^{4,38}$ Most interesting, the reverse analyses, in which gray and white matter volume differences were assessed while accounting for microstructural abnormalities, did not show significant regions of atrophy in patients with ALS compared with HCs, except for a small gray matter area in the right angular gyrus. This finding supports further the hypothesis that DTI indices have a higher sensitivity to ALS-related damage than volumetric measures. In addition, our findings did not change when we ran the analyses also accounting for CSF to minimize the partial volume effect (data not shown).

MD values of the orbitofrontal regions were significantly correlated with disease duration. This result is consistent with previous observations. ${ }^{39,40}$ Our failure to identify a correlation between $\mathrm{MD}$ and FA alterations and clinical disability is in keeping with previous data obtained from patients with ALS. ${ }^{41-44}$ In addition, in our study, patients had a relatively small spread of ALSFRS scores, and this might have worked against the ability to detect a correlation between structural changes and disability. We also observed MD changes in extramotor brain regions. This finding fits with the notion that ALS pathology does not spare extramotor cerebral areas and that, to a variable extent, it overlaps with the clinicopathologic features of frontotemporal lobar degeneration. ${ }^{45}$ Most remarkable, MD abnormalities were seen in structures that are typically hit by frontotemporal lobar degeneration, ${ }^{46}$ such as the insula, inferior frontal, orbitofrontal, and temporal regions.

This study is not without limitations. First, it is crosssectional, and we can only speculate that the observed microstructural alterations independent of volume loss may precede development of tissue atrophy. Second, as in other studies, ${ }^{12,33,39,44}$ we failed to find significant relationships between DTI abnormalities and clinical severity or progression rate. This is likely due to the relatively small size and the clinical homogeneity of the sample studied. Third, the small number of patients with bulbar onset did not allow us to assess the pattern of brain damage in these patients separately.

Finally, some methodologic issues related to MR imaging analysis should be addressed. The 2 scan modalities that we used differed in image resolution, and this difference may have affected the results. Nevertheless, because images were resampled to the same voxel size, smoothed equally, and rigidly aligned, we believe that such an impact was small, if any. The significance threshold for regional analysis was set at $P<.001$ or $<.005$, uncorrected for multiple comparisons, and such sensitive thresholds might have led to false-positive results. Although larger studies are needed to replicate our findings, we observed MR imaging changes only in brain areas that are known to be the preferential sites of ALS damage from neuropathologic studies. Finally, the relationship between volumes and MD/FA is complex, may be nonlinear, depends on the brain region studied, and may be affected by different pathophysiologic mechanisms. Therefore, by using a linear model, such as ANCOVA, we may not have captured the overall effect of volumetric changes on DTI differences and vice versa. 


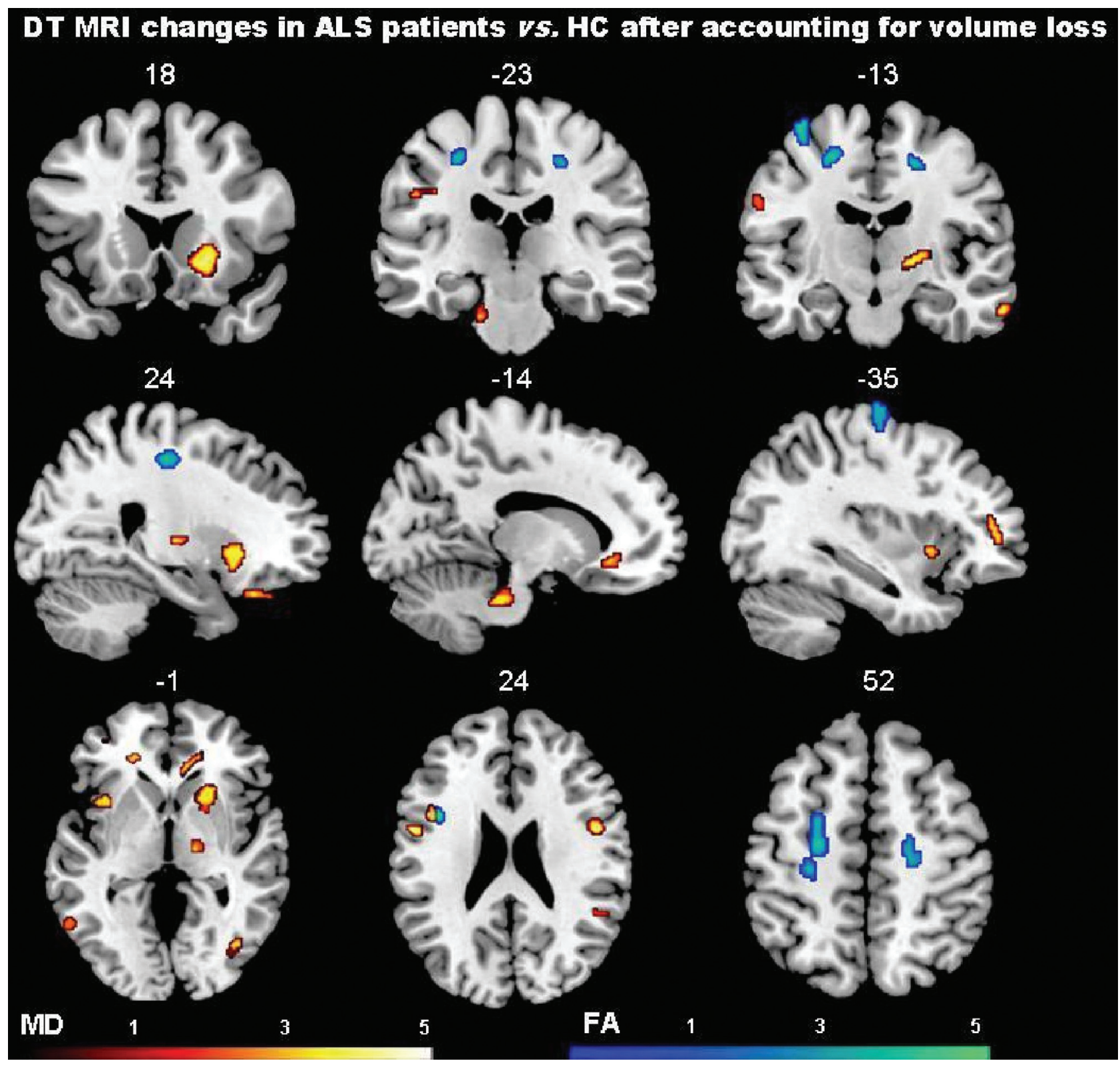

Fig 3. Regions of increased MD and decreased FA, after accounting for gray and white matter volume loss, in patients with ALS compared with HCs are shown on the same MNI template. Contrasts are shown at $P<.001$ uncorrected in at least 20 contiguous voxels. Color bars denote $t$ values. Results are in neurologic convention (right is right).

\begin{tabular}{|c|c|c|c|c|c|}
\hline \multirow[b]{2}{*}{ Cluster Size ${ }^{a}$} & \multirow[b]{2}{*}{ Regions } & \multicolumn{3}{|c|}{ MNI Coordinates $(\mathrm{mm})$} & \multirow{2}{*}{$\begin{array}{c}T \\
\text { Values }\end{array}$} \\
\hline & & $x$ & $Y$ & $\bar{Z}$ & \\
\hline 47 & L inferior frontal region & -41 & 10 & 22 & 5.71 \\
\hline \multirow[t]{2}{*}{133} & $\mathrm{~L}$ premotor area & -22 & -11 & 53 & 4.92 \\
\hline & $\mathrm{L}$ premotor area & -24 & -1 & 51 & 4.28 \\
\hline 91 & L precentral region & -38 & -15 & 69 & 4.63 \\
\hline \multirow[t]{2}{*}{150} & $\mathrm{R}$ premotor area & 19 & -15 & 50 & 4.44 \\
\hline & $\mathrm{R}$ precentral region & 24 & -20 & 48 & 4.34 \\
\hline \multirow[t]{2}{*}{56} & L precentral region & -29 & -24 & 50 & 4.17 \\
\hline & $\mathrm{L}$ precentral region & -29 & -16 & 50 & 3.53 \\
\hline
\end{tabular}

a The number of voxels. $P<.001$ uncorrected within at least 20 contiguous voxels.

\section{Conclusions}

We showed that microstructural changes affect various brain regions of patients with ALS and that they are independent of volume loss. It is, therefore, tempting to speculate that DTI alterations reflect loss of brain tissue integrity, which can lead to atrophy. Future longitudinal studies are needed to confirm this hypothesis and clarify whether DTI can be useful in making an earlier diagnosis in patients suspected of having ALS. 
Disclosures: Federica Agosta belongs to the Speaker Bureau for Bayer Schering. Giancarlo Comi is a consultant for Bayer Schering and Merck Serono and has financial relationships with TEVA Pharmaceutical Industries Ltd, Novartis, Merck Serono, the Serono Symposia International Foundation, Bayer Schering, and Biogen Dompé. He received an honorarium for speaking activities. Massimo Filippi received research support (including provision of equipment and materials) from Bayer Shering, Biogen Dompé, Genmab A/S, Merck Serono, TEVA Pharmaceutical Industries Ltd, Fondazione Italiana Sclerosi Multipla, and Fondazione Mariani. He belongs to the Speaker Bureau for Bayer Shering Pharma, Biogen-Dompé, Genmab A/S, Merck Serono, and TEVA Pharmaceutical Industries Ltd. He is a consultant for Bayer Shering Pharma, Biogen-Dompé, Genmab A/S, Merck Serono, and TEVA Pharmaceutical Industries Ltd.

\section{References}

1. Hughes JT. Pathology of amyotrophic lateral sclerosis. Adv Neurol 1982;36:61-74

2. Turner MR, Kiernan MC, Leigh PN, et al. Biomarkers in amyotrophic lateral sclerosis. Lancet Neurol 2009;8:94-109

3. Rothstein JD. Current hypotheses for the underlying biology of amyotrophic lateral sclerosis. Ann Neurol 2009;65(suppl 1):S3-9

4. Pierpaoli C, Jezzard P, Basser PJ, et al. Diffusion tensor MR imaging of the human brain. Radiology 1996;201:637-48

5. Basser PJ, Mattiello J, LeBihan D. Estimation of the effective self-diffusion tensor from the NMR spin echo. J Magn Reson B 1994;103:247-54

6. Agosta F, Chio A, Cosottini M, et al. The present and the future of neuroimaging in amyotrophic lateral sclerosis. AJNR Am J Neuroradiol 2010;31:1769-77 Epub 2010 Apr 1

7. Agosta F, Pagani E, Rocca MA, et al. Voxel-based morphometry study of brain volumetry and diffusivity in amyotrophic lateral sclerosis patients with mild disability. Hum Brain Mapp 2007;28:1430-38

8. Ciccarelli $\mathrm{O}$, Behrens TE, Johansen-Berg H, et al. Investigation of white matter pathology in ALS and PLS using tract-based spatial statistics. Hum Brain Mapp 2009;30:615-24

9. Sach M, Winkler G, Glauche V, et al. Diffusion tensor MRI of early upper motor neuron involvement in amyotrophic lateral sclerosis. Brain 2004; 127:340-50

10. Sage CA, Peeters RR, Gorner A, et al. Quantitative diffusion tensor imaging in amyotrophic lateral sclerosis. Neuroimage 2007;34:486-99

11. Sage CA, Van Hecke W, Peeters R, et al. Quantitative diffusion tensor imaging in amyotrophic lateral sclerosis: revisited. Hum Brain Mapp 2009;30:3657-75

12. Thivard L, Pradat PF, Lehericy S, et al. Diffusion tensor imaging and voxel based morphometry study in amyotrophic lateral sclerosis: relationships with motor disability. J Neurol Neurosurg Psychiatry 2007;78:889-92

13. Salat DH, Tuch DS, Hevelone ND, et al. Age-related changes in prefrontal white matter measured by diffusion tensor imaging. Ann N Y Acad Sci 2005;1064:37-49

14. Chang JL, Lomen-Hoerth C, Murphy J, et al. A voxel-based morphometry study of patterns of brain atrophy in ALS and ALS/FTLD. Neurology 2005;65:75-80

15. Grosskreutz J, Kaufmann J, Fradrich J, et al. Widespread sensorimotor and frontal cortical atrophy in amyotrophic lateral sclerosis. BMC Neurol 2006;6:17

16. Kassubek J, Unrath A, Huppertz HJ, et al. Global brain atrophy and corticospinal tract alterations in ALS, as investigated by voxel-based morphometry of 3-D MRI. Amyotroph Lateral Scler Other Motor Neuron Disord 2005;6:213-20

17. Kato S, Hayashi H, Yagishita A. Involvement of the frontotemporal lobe and limbic system in amyotrophic lateral sclerosis: as assessed by serial computed tomography and magnetic resonance imaging. J Neurol Sci 1993;116:52-58

18. Roccatagliata L, Bonzano L, Mancardi G, et al. Detection of motor cortex thinning and corticospinal tract involvement by quantitative MRI in amyotrophic lateral sclerosis. Amyotroph Lateral Scler 2009;10:47-52

19. Abrahams S, Goldstein LH, Suckling J, et al. Frontotemporal white matter changes in amyotrophic lateral sclerosis. J Neurol 2005;252:321-31

20. Ellis CM, Suckling J, Amaro E Jr, et al. Volumetric analysis reveals corticospinal tract degeneration and extramotor involvement in ALS. Neurology 2001;57:1571-78

21. Hugenschmidt CE, Peiffer AM, Kraft RA, et al. Relating imaging indices of white matter integrity and volume in healthy older adults. Cereb Cortex 2008; $18: 433-42$

22. Canu E, McLaren DG, Fitzgerald ME, et al. Microstructural diffusion changes are independent of macrostructural volume loss in moderate to severe Alzheimer's disease. J Alzheimers Dis 2010;19:963-76

23. Casanova R, Srikanth R, Baer A, et al. Biological parametric mapping: a statistical toolbox for multimodality brain image analysis. Neuroimage 2007; 34:137-43

24. Brooks BR. El Escorial World Federation of Neurology criteria for the diagnosis of amyotrophic lateral sclerosis: Subcommittee on Motor Neuron Diseases/Amyotrophic Lateral Sclerosis of the World Federation of Neurology Research Group on Neuromuscular Diseases and the El Escorial "Clinical limits of amyotrophic lateral sclerosis" workshop contributors. J Neurol Sci 1994;124(suppl):96-107

25. Cedarbaum JM, Stambler N, Malta E, et al. The ALSFRS-R: a revised ALS functional rating scale that incorporates assessments of respiratory functionBDNF ALS Study Group (Phase III). J Neurol Sci 1999;169:13-21

26. de Carvalho M, Scotto M, Lopes A, et al. Clinical and neurophysiological evaluation of progression in amyotrophic lateral sclerosis. Muscle Nerve 2003;28:630-33

27. Turner MR, Cagnin A, Turkheimer FE, et al. Evidence of widespread cerebral microglial activation in amyotrophic lateral sclerosis: an [11C](R)-PK11195 positron emission tomography study. Neurobiol Dis 2004;15:601-09

28. Ashburner J. A fast diffeomorphic image registration algorithm. Neuroimage 2007;38:95-113. Epub 2007 Jul 18

29. Ashburner J, Friston KJ. Unified segmentation. Neuroimage 2005;26:839-51

30. McLaren DG, Kosmatka KJ, Kastman EK, et al. Rhesus macaque brain morphometry: a methodological comparison of voxel-wise approaches. Methods 2010;50:157-65. Epub 2009 Oct 31

31. Horsfield MA. Mapping eddy current induced fields for the correction of diffusion-weighted echo planar images. Magn Reson Imaging 1999;17:1335-45

32. Graves MC, Fiala M, Dinglasan LA, et al. Inflammation in amyotrophic lateral sclerosis spinal cord and brain is mediated by activated macrophages, mast cells and T cells. Amyotroph Lateral Scler Other Motor Neuron Disord 2004;5:213-19

33. Agosta F, Rocca MA, Valsasina P, et al. A longitudinal diffusion tensor MRI study of the cervical cord and brain in amyotrophic lateral sclerosis patients. J Neurol Neurosurg Psychiatry 2009;80:53-55

34. Cheung G, Gawel MJ, Cooper PW, et al. Amyotrophic lateral sclerosis: correlation of clinical and MR imaging findings. Radiology 1995;194:263-70

35. Goodin DS, Rowley HA, Olney RK. Magnetic resonance imaging in amyotrophic lateral sclerosis. Ann Neurol 1988;23:418-20

36. Hecht MJ, Fellner F, Fellner C, et al. MRI-FLAIR images of the head show corticospinal tract alterations in ALS patients more frequently than T2-, T1and proton-density-weighted images. J Neurol Sci 2001;186:37-44

37. Hecht MJ, Fellner F, Fellner C, et al. Hyperintense and hypointense MRI signals of the precentral gyrus and corticospinal tract in ALS: a follow-up examination including FLAIR images. J Neurol Sci 2002;199:59-65

38. Pierpaoli C, Barnett A, Pajevic S, et al. Water diffusion changes in Wallerian degeneration and their dependence on white matter architecture. Neuroimage 2001;13:1174-85

39. Ellis CM, Simmons A, Jones DK, et al. Diffusion tensor MRI assesses corticospinal tract damage in ALS. Neurology 1999;53:1051-58

40. Wang S, Poptani H, Woo JH, et al. Amyotrophic lateral sclerosis: diffusiontensor and chemical shift MR imaging at 3.0 T. Radiology 2006;239:831-38

41. Ciccarelli O, Behrens TE, Altmann DR, et al. Probabilistic diffusion tractography: a potential tool to assess the rate of disease progression in amyotrophic lateral sclerosis. Brain 2006;129:1859-71

42. Mitsumoto H, Ulug AM, Pullman SL, et al. Quantitative objective markers for upper and lower motor neuron dysfunction in ALS. Neurology 2007; 68:1402-10

43. Schimrigk SK, Bellenberg B, Schluter M, et al. Diffusion tensor imaging-based fractional anisotropy quantification in the corticospinal tract of patients with amyotrophic lateral sclerosis using a probabilistic mixture model. AJNR Am J Neuroradiol 2007;28:724-30

44. Toosy AT, Werring DJ, Orrell RW, et al. Diffusion tensor imaging detects corticospinal tract involvement at multiple levels in amyotrophic lateral sclerosis. J Neurol Neurosurg Psychiatry 2003;74:1250-57

45. Phukan J, Pender NP, Hardiman O. Cognitive impairment in amyotrophic lateral sclerosis. Lancet Neurol 2007;6:994-1003

46. Rosen HJ, Gorno-Tempini ML, Goldman WP, et al. Patterns of brain atrophy in frontotemporal dementia and semantic dementia. Neurology 2002; 58:198-208 